\title{
SyTroN: A Virtual Classroom for Collaborative and Distant E-Learning System by Teleoperating Real Devices
}

\author{
R. Chellali, ${ }^{1}$ C. Dumas, ${ }^{2}$ Nicolas Mollet, ${ }^{1}$ and G. Subileau' \\ ${ }^{1}$ TERA Department, Italian Institute of Technology, Via Morego, 30. Genova 16163, Italy \\ ${ }^{2}$ Ecole des Mines de Nantes- 4 rue a. Kastler, 44000, Nantes, France \\ Correspondence should be addressed to R. Chellali, ryad.chellali@iit.it
}

Received 11 September 2008; Revised 2 March 2009; Accepted 30 March 2009

Recommended by Abdennour El Rhalibi

Distant E-learning is a main issue nowadays, and it is strongly motivated by social and economical considerations. The increased people mobility and the reduction of educational costs push to develop ad hoc solutions enabling to access to knowledge regardless to geographical situation and economical capabilities. These parameters should not be limits for good training: learning material's pertinence and efficiency have to remain the core of educational activities. In this paper we address the problem through SyTroN: a tele-learning system. This system combines virtual reality and teleoperation techniques to offer an open platform with two main objectives. The first one is to propose intuitive virtual classrooms/desks, including a real teacher supervision and supporting collaborative and individual distant learning. The second goal is to place learners in real conditions with remote connections to real devices allowing distant experimentations. Both goals participate to increase learning impacts and to reduce costs, that is, sharing costly real devices from anywhere at any time. After 5 years of development, our work has been validated by an extensive use at a high engineering school. In situ tests and learning impact studies have been done. They show some advantages and some drawbacks of our global solution.

Copyright ( $) 2009$ R. Chellali et al. This is an open access article distributed under the Creative Commons Attribution License, which permits unrestricted use, distribution, and reproduction in any medium, provided the original work is properly cited.

\section{Introduction}

E-learning is nowadays well established as a promising education vector. Thanks to the growth of computer-based technologies (including Internet, video games, virtual reality, etc.), classical education started to shift since early 1990s from old fashion classrooms to virtual collaborative spaces and other mobile-learning devices [1]. Industrialists were the first to investigate in this area: they designed tailored and huge prototyping, simulation, and training systems. The VTT project, for instance, was developed to train people on how to execute technical gestures while manipulating a milling machine [2]. We can also cite the GVT project [3], with individual and collaborative [4] environment learning of industrial maintenance procedures on military equipment.

This movement was strongly amplified by popularization of both Internet and virtual reality technologies. The first one is by offering affordable networking facilities, and the second one is by enabling the use of low-cost, attractive, and useful learning environments. Text-based contents were used in the beginning. Multimedia contents and also realistic and complex interactive environments were developed to support the learning process. This became possible because the techniques were mature, and the costs have been largely reduced: powerful and cheap computers were largely spread enabling advanced and multimodal interactions. We have also to notice that video games also played a major role. Indeed, the gaming market pushed VR technologies like 3D rendering hardware and interfaces to make them affordable (the Nintendo Wii is a good example of this success). The conjunction of these factors allowed virtual classroom metaphors to exist $[5,6]$.

Many distant learning platforms are now in use [7] in many areas. Unfortunately, most of existing systems do not support tangible contents. Only text, audio-visual, and simulations are available. For engineering disciplines, intangible, predefined, and simulation-based contents are necessary but not sufficient to transmit the knowledge: experiencing real world and real situations is mandatory to 
have the maximum impact [8]. Indeed, the essence of engineering is to build abstractions representing the real world in order to deal with it. Consequently, the corresponding learning techniques start generally by simplified models and describing physical phenomena. Solutions to study these lasts are then propose. Unfortunately, one can only simulate the phenomena (through virtual reality and predefined models) and cannot fully integrate the reality with its errors and uncertainties. Accessing to physical objects is then necessary. Following that, Balestrino et al. [9] addressed automatic control field and built a system to learn the usage of a robotic arm [10]. Kim et al. [11] has focused on simulating electronic circuits in a distant virtual laboratory with the ability to apply the models on real equipment. He included some feedbacks, mainly the use of webcams, to visualize and validate theoretical simulations.

Other developments have been achieved to enable connecting more complex systems, namely, systems with high dimensionality (e.g., MIMO, multiinput multioutput systems like mobile robots) but with limited capabilities. These limitations are not technical but conceptual. Indeed, usability of e-learning systems (i.e., how easy is the integration of new topics-lectures) is generally neglected. For instance, some developed infrastructures are mostly one-shot systems (where adding any new function or any new service needs consequent efforts). Other systems use proprietary softwares (namely, closed systems like COSIMIR Robotics) or have very poor interfaces leading to cognitive overloads and so are not dealing with e-learning principles $[12,13])$.

Another philosophy for increasing the learning process impact was introduced in the early 2000s. It is concerned with so-called serious games (see the serious game initiative at http://www.seriousgames.org/). Initiated for military application and extend for other fields (health, rescue, economy, etc.), its aim is to help the creation of simulations having the look and the feel of classical games but concerned with real-world situations, processes, or events. This is not really far from edutainment philosophy and can help to improve it. Namely, one needs to investigate whether or not adding playful activities is of interest.

When revisiting the last mentioned principles, one can derive the factors affecting the learning impact. They are three, and many science of education researchers [14-16] consider them as key points to qualify an efficient e-learning system.

(i) Practice. The level of realism and the richness of the system let learners confronted with realities and real situations.

(ii) Sharing-out. The available means for communications and for exchanges within the system allows learners to enhance team working and to improve communication skills $(80 \%$ of the engineer real life workload is concerned with team working, management, and communication).

(iii) Presence. How immersion (seen from the VR point of view) learners feel within the system lets their attention and awareness turned to skill acquisition [17].
Following the previous constraints and considerations, we started the SyTroN project. The project aim was not only to tackle educational aspects but also to consider technical solutions offering an open and a complete solution. Namely, we integrate and we develop technologies to built a system that

(1) supports the classroom metaphor dealing with educational needs,

(2) is enough opened enabling operational flexibility,

(3) is enough intuitive (from back-office and front-office sides) allowing to not accustomed users to use the system, and

(4) can be run on trivialized support (both hardware and software) with no specific needs.

Innovative solutions by mixing virtual reality, networking and teleoperation technologies were developed. With these techniques, we achieved a complete and functional solution allowing currently training on 3 different physical devices. Learners can follow a two-step process: first, they learn by simulation (acquiring theoretical skills), and then they manipulate real devices and solve real problems.

The first section of this paper deals with the presentation of models and implementations of SyTroN components. The second section gives an overview of the system usage and some e-learning session examples. The third section presents the field validation of SyTroN, evaluations, and some perspectives for the future developments.

\section{SyTroN's Model and Implementation}

In this section we present the models and the implementations we did to set up our system. Mainly, we adapted existing solutions to reach our goal. We adopted the classical scheme where e-learning systems are divided into three logical units: users, knowledge database, and learning environments. These units are connected and combined to deliver material and to offer optimal learning conditions. We first list the situations and activities any user may face during a session. From that, we derive corresponding solutions regarding three axes, namely, (1) connectivity allowing to logical units to be linked and to exchange information, (2) the working environment offering a virtual desk (personal space) or a classroom (a shared space) to learners, and (3) the interaction tools letting learners interact with both virtual and real worlds.

2.1. Pedagogy Design Considerations. Before beginning the development of SyTroN and writing its codes, we made some practical investigations, and we interviewed all the participants. Teachers were asked to detail their presence-based lectures regarding structural aspects, timing, and exchanges (teacher-students, students-students) during lectures. We asked students how they perceive the training sessionsas well. This study leads us to derive a general framework. We worked on a script with handmade screens and concepts of what should be the virtual desk and the virtual classroom. 


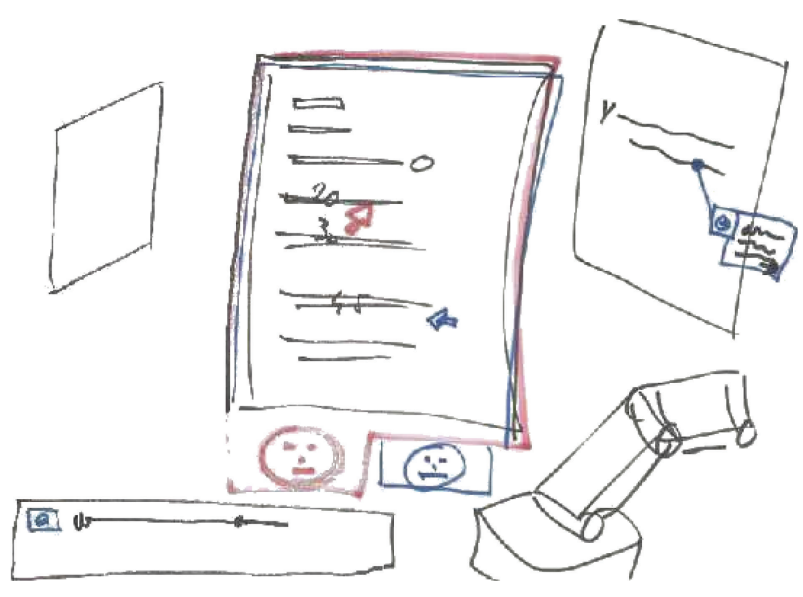

FIGURE 1: Hand drawings of workspace's prototype.

Iteratively, we refined the models to reach an acceptable frame. Our approach is not only objective: some of our considerations are purely subjective to take into account personalities of teachers and how they transmit knowledge. Students also evaluate the perceived quality of knowledge transmissionas well. They showed more sensitivity to the way the teacher introduces and explains concepts than the validity of concepts. We found out a big variability in both groups: teaching schemes and their perception by receivers are personality dependant.

Teachers Interviews. The goal in interviewing teachers is to have a generic description of sessions' progress. Three points were considered.

(i) Timing. We asked teachers to describe the training sessions phases. It turned out that most of them divide sessions in three periods. The first one is concerned with offline preparation: students must acquire the theoretical knowledge and understand the training session progress, that is, the learning steps. The second period is the session itself, and the last one is dedicated reporting.

(ii) Interactions. During a session: teachers interact with students depending on situations; they are "invasive" to steer the work or "passive" to let students develop their autonomy.

(iii) Reporting and evaluation. A lot of practices are existing. Some teachers prefer a predefined reporting process to evaluate students while others let students free to produce a self-assessment.

Students Interviews. We wanted here to extract the students' optimal imaginary working environment and sessions progress. The main request coming from interviews was the flexibility. Their aim is a system accessible at any time, from anywhere, allowing them to share the work with others in a dynamic and an easy way, a working environment where all the needed materials are quickly useful, and so on.
The interviews were for us a good support to derive the SyTroN framework. Figure 1, for instance, is a draft made by students to describe the personal workspace. Collaborative, interactive aspects as well as capabilities to face real situations were the key points we addressed to deal with users' expectations.

2.2. E-Learning Situations and Activities. To launch the effective development, we started by listing situations and activities within e-learning system. To do so, we considered potential learning session scenario.

(1) User Connection. A teacher or a student requests a connection. After verifying the rights from the knowledge database, the user is given the access to a list of actions he or she is able to do.

(2) Standalone Activities. Once connected, the user works alone. He or she accesses the personal working space. User can thus execute synchronous $(3,5)$ or asynchronous activities $(3,4)$.

(3) Working with Other Users. In addition to what is possible to do in standalone mode, users are allowed to connect to a working group. Additional exchanges may concern chatting with other users, having a videoconference with them, or just sending emails.

(4) Getting or Uploading Material. Users can download lectures and contents. It could be text-based, audio, or video materials. Email and messages can be also exchanged. On the other hand, teachers or administrators can upload material.

(5) Using Devices. A user can interact with a virtual or a physical device in real time. This is the main sensitive action a user can perform. Following the availability of the device and the user's rights, a device is allowed to one user (except when the device is shared between the teacher and the student). The interaction here is mainly synchronous and time critical because the control loop is not local, but it is geographically distributed over the network.

2.3. Developed Infrastructure. To support the previous activities and situations, we designed and deployed a fully connected network. This network supports all client-servers'based services and functions the users need. Figure 2 illustrates SyTroN's network. This network allows multiple and distant IT-based connections with a main goal: ensure flexibility. Indeed, SyTroN handles connections regardless users' geographical distribution as well as material's distribution allowing to several institutions to access to the system 24 hours a day around the world. Hereafter we detail SyTroN functions and services. 
SyTroN supports functions and offers services that can be divided into three categories:

(i) user oriented (including teachers and students),

(ii) device oriented, and

(iii) management oriented.

User Oriented Services and Functions. Two families of functions were implemented: communication functions and device control functions. The first ones are classical in elearning system. The second ones are more specific; they let users control remotely physical devices.

Communication functions enable users to access to the contents and to people within the SyTroN system. Users can

(i) access to lectures, tests, and evaluations,

(ii) contact teachers and other students,

(iii) get the devices status and gateways,

(iv) simulate devices and processes.

Some specific functions are reserved to teachers like adding devices, adding lectures, and setting tests and evaluations.

Device control functions link users to physical devices. These functions achieve remote physical interactions by sending controls and receiving measurements in return, namely, device status and partial information about the remote environment.

Device Oriented Services and Functions. These functions are gateways between users (device control functions) and the remote physical world. The functions set is composed of two parts: the device gateway and the device controller.

The device gateway connects the users and the knowledge database to the device. The device controller pilots the actuators and gets the remote sensors information. The device gateway is just a translator. Depending on the considered device, the gateway formats the users commands and passes the parameters to the controller. The concerned actuators execute the commands and respond to the device controller by giving the new status (e.g., position of a motor). In other words, we use a remotely closed-control scheme. Thus, the gateway functions are mainly dealing with synchronous and critical communication. TCP and acknowledged UDP protocols are used to guaranty the exchanges synchronicity and integrity.

The device controller is the embedded interface between real and the logical world (the device gateway). Its main function is to close the local low level control loop. This component is device-dependent: each device utilizes its own specific driver. For SyTroN implementation, three devices with three different dynamics have been tested. Due to the nature of these devices, two control platforms are used: the Matlab-Simulink (from MathWorks) platform and a proprietary platform (C++-based API). Both support real time controls. Indeed, for the two, we have compiled binaries connectable and executable at a high-frequency rate $(100 \mathrm{~Hz})$ compatible with control constraints (10 milliseconds to control systems with time constant of 100 milliseconds and more).

Administration and Management Services and Functions. The knowledge database (K-database) contains all the information needed to manage the global system. Four subdatabases describe the logical units (teachers, students, lectures, and devices) and their cross-relationships. The main functions of the K-database are the following.

(i) Managing people by setting and verifying the rights of users such as name, coordinates (email, address), list of lectures. Each field allows users to access to specific SyTroN services.

(ii) Managing contents such as Lectures, tests, and evaluation program and tests and evaluation results.

(iii) Managing devices by adding, modifying, or keeping devices histories. Each device is described as a MIMO system, with its inputs and outputs (current user, users priorities, IP addresses and ports, data formats, scales, intervals, ect.).

This relational database is SQL compliant (MySQL-PHP based). For instance, the devices' table is related to lectures. Through this, a student connection will extract the list of the allowable lectures, let him choose one of them, and then access to the targeted device. Once connected, the student load the device characteristic parameters. The student can thus start his interactions.

Within the K-Base, we added a gateway module to open partly the system to let the engineering school administration to program teaching activities like scheduling, compiling results, establishment of appointments, and so on.

\section{The Virtual Desk and the Working Environment}

In the previous section, we described the set of functions SyTroN supports. In this section we show how these functions are materialized through a VR-based interface. The main purpose here is to immerse the user within practical situations to let him view, perceive, and become aware of the learning world (the teacher, the other learners and the learning object) with a minimum cognitive workload: no additional efforts are requested to imagine other participants or controlled devices for instance. We worked on two main aspects: the personal working space and the shared environment. The first aspect is concerned with the design of a functional personal space allowing intuitive and effortless activities. The second aspect is more dedicated to collaborative activities. The challenging task here was to built a unique " $P C$ window" knowing that both have to be represented on it. Needless to say that the playful aspect was hidden in our design. Nevertheless it was constantly in our mind at least in representing the working space environment. 


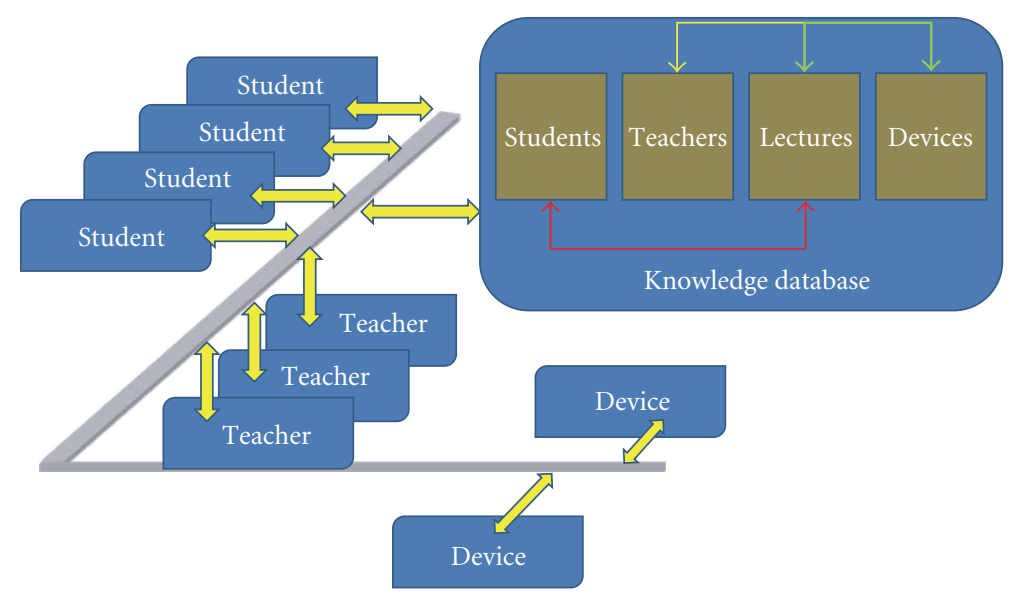

FIgURE 2: Global architecture of SyTroN.

3.1. Modeling the Workspace. The personal workspace is the place where the learner must feel as if he or she were on a desk with accesses to some of previously introduced functions. The model we choose emphasizes the immersion, the easy navigation, and the awareness of the other activities and offers several modes to control the devices.

Immersion and Awareness. The materialization of this metaphor is 3D-based: this choice is very useful because it increases immersion, and let us distribute spatially the learning objects. Indeed, the current object of interest is central while active and nonactive objects are placed on side walls. This let the user focus on the central object while keeping access to other functions. in Figure 3, a device is in the first plane, and documents for instance are on the topright side.

Navigation and Access to Services. The 3D approach eases the navigation process. Indeed, by clicking on a service, the corresponding representation is brought forward. In Figure 4, the user is consulting a document from the library. One can notice that other functions and tools are still visible.

Manipulating the Devices and Interacting with the Real World. Manipulating real devices is one of the key points of our system. Students perform first a theoretical phase (e.g., working on documents). After that, the learner can start controlling the device. This can be done

(1) in a direct way by accessing directly to the device parameters in an open-loop mode;

(2) in a high-level way by programming an off-line trajectory (a set of values or time dependant functions);

(3) in a low-level way by setting up the remote control loop.

For each type of control, the user has three possibilities: manipulating directly the device buttons, running a simulation which generates the desired data(the trajectory in the parameters' space), or setting up a controller.
To increase the presence feeling, we added a live video feedback of the real device (see Figure 5). Indeed, we noticed that learners were frustrated by having only a virtual representation of the manipulated devices, specially for the mobile robot. When programming an obstacle avoidance procedure, learners verified twice the procedure before running it: they feel responsible of the robot. This reaction is to be considered as an impact learning increasing.

3.2. Shared Space. The class room metaphor was our guide to extend the personal workspace to offer a collaborative platform to support the groupwork. This aspect is mainly social and is concerned with communication between users [18]. Following that, we added some specific items like avatars and multimodal services like telepointers and verbaltext channels.

Self-Representation and Copresence. We made the hypothesis that feeling belonging to a team starts by feeling the copresence of other team members. To do so, each member of the session is represented by an exocentric avatar. When a user focuses on a service, his avatar in all other students' interface flies next to the object corresponding to this service, in order to indicate to the other participants what he is doing.

Multimodal Communication between Users. For SyTroN we used a communication server named Reflector. Reflector supports a set of Peer-to-Peer or broadcast-like verbal and textual exchanges like chatting or voice conferencing. To address nonverbal communication, we added a virtual white board to let users share results and formula through drawings thanks to a pen tablet or mouse. Also and for the same purposes, we added the telepointer: it is the duplication of one's pointing device position on the other personal spaces. It can tell partners what action is targeted.

Sharing a Same Device. To simplify the architecture of the system, we assume that the use of any device is exclusive: only one user can send controls and set control parameters 


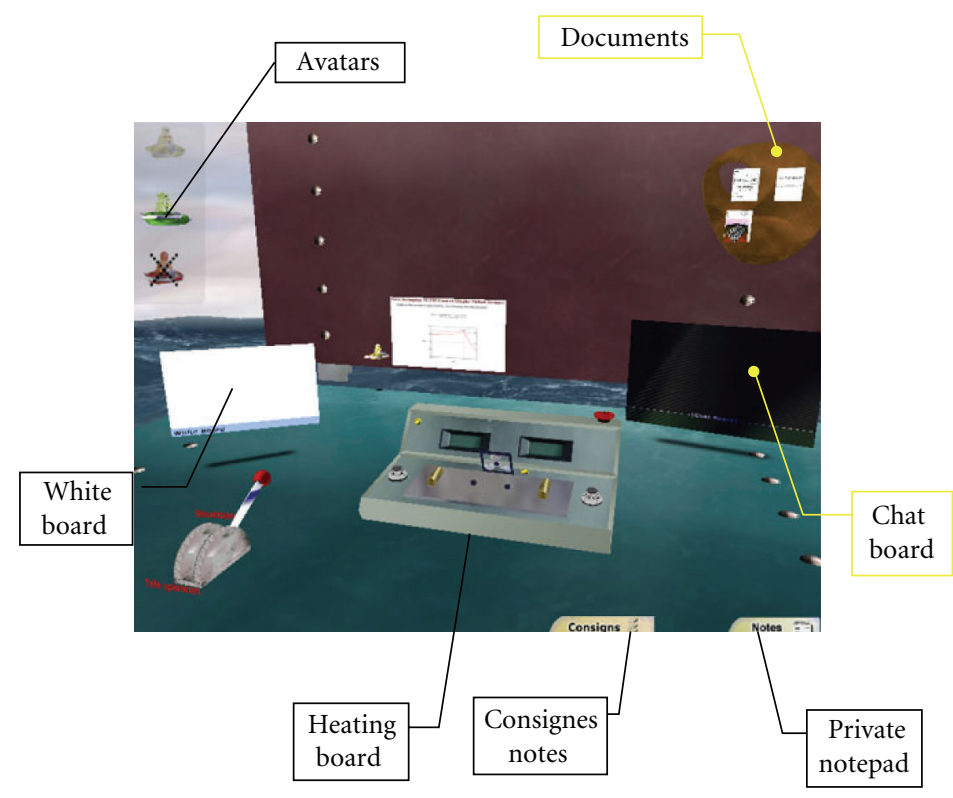

FIgURE 3: The personal workspace.

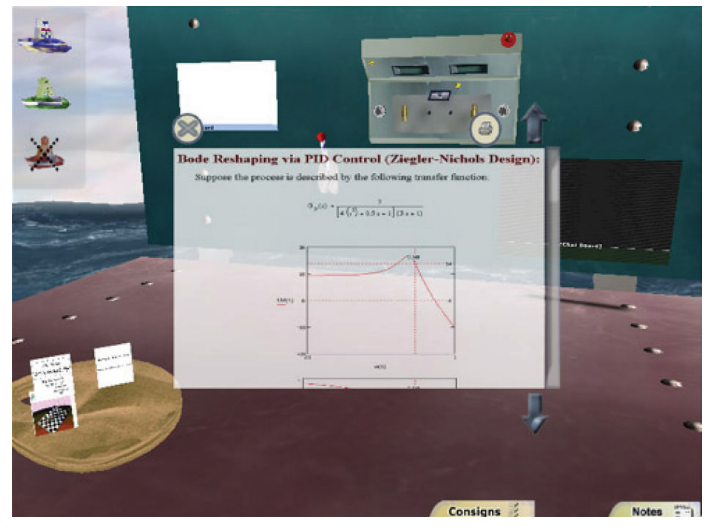

FIgURE 4: Navigation to access to services.

to the device. On the contrary, one or more participants can observe the device by receiving its status. Following that and to avoid conflicts, we use a priority-token policy: each device is represented by a unique token, taking this last is equivalent to take the device control. On the other hand, each user has a level of priority defined within the K-Base. A user with level $n$ can interrupt any current device user having a lower priority level. In practice, users communicate through verbal or textual channels to solve conflicts.

\section{SyTroN Usage through Examples}

We give in this section a short description of two devices we have integrated in SyTroN and some views of the running system.

4.1. Devices. The figure presents two of the three devices we have already included in our system.

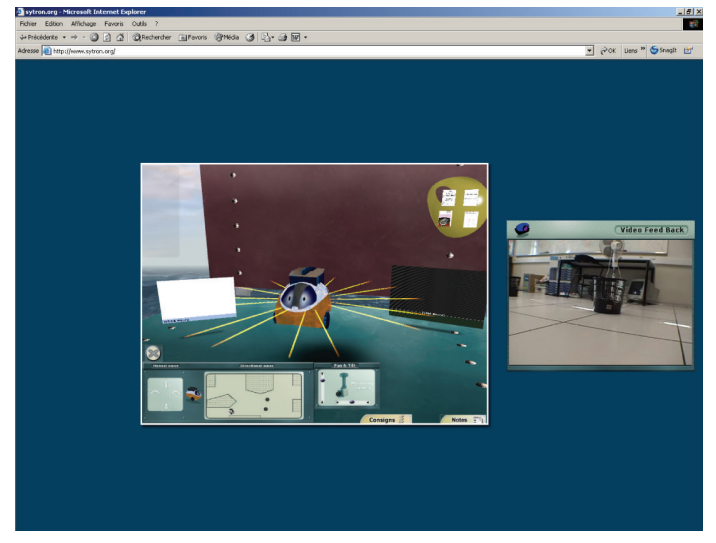

FIGURE 5: Video feedback service.

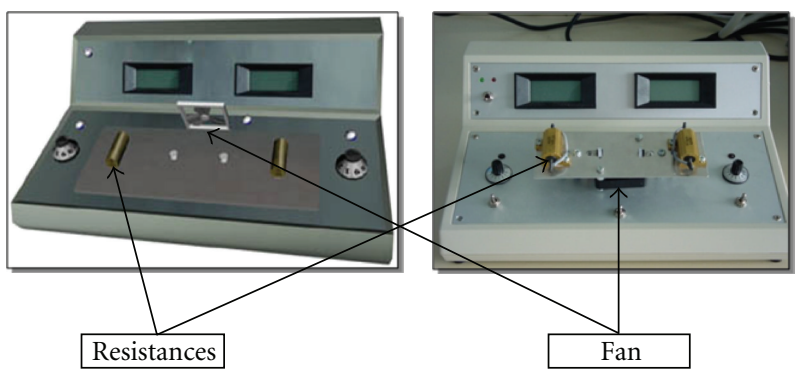

Figure 6: The heating board.

The heating board (Figure 6) is dedicated to study the heat propagation processes. It is a good example to learn how to control complex systems with numerous inputs and outputs in the state space (an algebraic approach of automatic control). The heating board is a basic Multiple Inputs/Multiple Outputs system (MIMO). The students 


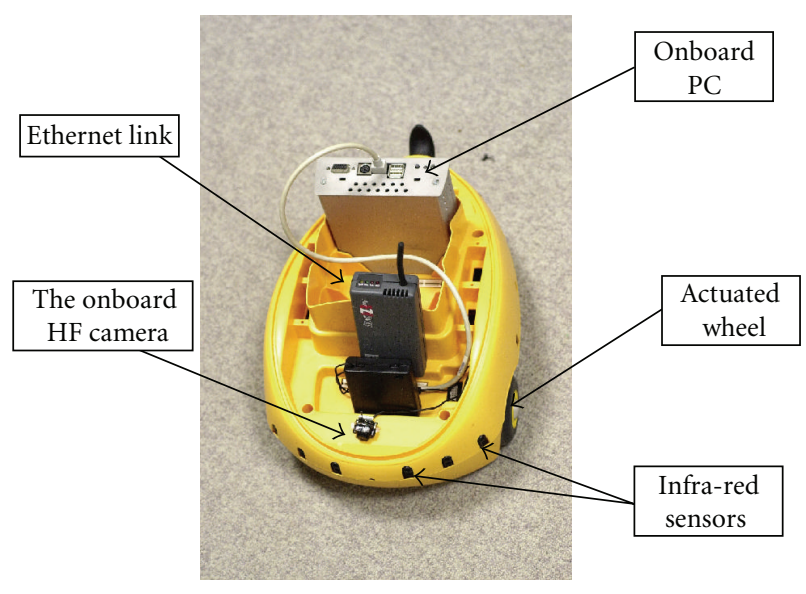

Figure 7: The Pekee robot.

have here to keep the temperature of the board constant, regardless to the external temperature (considered here as a perturbation). The goal of the training session is to learn MIMO control techniques, especially control laws.

The mobile Pekee robot (Figure 7) is a classical robot produced by Wany Robotics SA. This robot has two actuators, namely, two DC motors enabling the robot to move with 3 degrees of freedom. On the other hand, Pekee has onboard a set of sensors (IR telemetry, light, gyros, choc detector, etc.) enabling to evaluate the robot's environment. Both actuators and sensors are handled by the onboard PC. This last communicates with any other PC using a wireless Ethernet channel. The communication can concern the state of the robot (sensors information) or robot controls (DC motors position/speed or a request for a specific measurement).

4.2. Usage. Starting the session, the student downloads the documents explaining the lecture, the manipulation protocol, and the attended results. This first step is followed by the simulation phase: the learner can start training on the virtual device (equivalent of the real one) in a standalone mode or within a group. This enables to acquire the theoretical basis. After that, the student(s) can pass to real manipulation regarding the given protocol as specified in the lecture documents.

The Heating Board. The student can verify the results of the simulations by comparing resulting curves to theoretical ones. From the derived model, the student can switch to the real device to set up the control parameters, namely, the PID constants. These parameters are sent via the asynchronous TCP channel to the device server, and the real test can start. The closed loop control is then activated to maintain the temperature to a desired one. The client calculates the inputs of the heating board and sends it via the TCP synchronous channel. The device server extracts the current temperature and returns it to the client and so on. This closed-loop process is done at $1 \mathrm{~Hz}$ frequency. Indeed, as the response time of the system is greater than 15 seconds, $1 \mathrm{~Hz}$ frequency for the control loop is enough to verify the Shanon theorem and to ensure the stability of the system.

The Mobile Pekee Robot. The mobile robot is an example of nonlinear systems. Due to its nonholonomy, learners must develop advanced control strategies to execute some simple tasks like trajectory following or obstacle avoidance. The first step is to aware students about time constraints. Here, these constraints are stronger than those of the previous device. Indeed, the purpose here is to enable to a distant student to pilot a mobile. It is obvious that the shorter the system response time is, the better the manipulation is. An ACK-UDP-based protocol is used for this service. As for the heating board, the first stages of the mobile robot manipulations are concerned with the discovery of the device. This is done using documents and simulations. For the teleoperated mode, the user may use a force feedback joystick, a mouse, or the computer keyboard and the visual feedback (a video stream coming from the embedded camera) to pilot the robot. This generates motion commands that are sent to the robot. The robot executes these commands, captures about 31 sensors measurements, and replies to the client. These measurements are then used to refresh the virtual environment. As the robot speed is about $0.5 \mathrm{~m} / \mathrm{s}$, the user may react in less than $1 / 2$ second to avoid obstacles in front of the robot. This response time is obtained by tuning the range sensor dynamics to $1 \mathrm{~m}$ depth. Following that and considering transmissions time delays in the network, any object about $0.25 \mathrm{~m}$ has to be considered as very close obstacle. A hidden mobile robot was added to the interface to take into account the effects of time delays (due mainly to the network) and the resulting offset between the user command and the real state of the remote robot. Once the discovery phase finished, students write high-level controllers (polygonal approximation-based or BUG-2 like strategies) to execute a robot displacement.

\section{Evaluation}

The evaluation of the system has been done to measure two quantities: the perceived quality and the learning impact factor. The first quantity is well known in human-machine interface and is subjective. Its role is to determine the system's usability degree. Classically the evaluation of such degree is done through questionnaires and psychophysics studies. The second quantity is more objective and can measure how efficient is the system as a vector for knowledge and skills acquisition. This evaluation was done by comparing the results obtained by learners in tests in both classical learning process and the SyTroN-based one.

5.1. The Perceived Quality. For this evaluation, we agreed with all participants to consider three parameters.

(1) The personal workspace quality.

(2) Collaborative facilities efficiency.

(3) The realism of the interaction with the real world. 
These parameters reflect more or less participants' aims expressed during interviews. For the evaluation protocol, we decided to work with two groups: a group following the normal program for young engineer and a second one following the adult program. For this last, students work mainly from home and nightly. For each group we evaluated the three parameters, and questionnaires were filled.

The Workspace Quality. Users were asked to work alone and to try to discover all SyTroN functionalities. Individual sessions were performed by participants. We verify our preconception hypothesis: the interface is enough intuitive and users discovered services in a quasinatural way. Users verified that all metaphors concerning a generic personal workspace are present. They verified also that the navigation between the subspaces (documents, manipulations, communications, etc.) is easy: the subspaces are simultaneously present within the $3 \mathrm{D}$ representation and with a simple mouse click one can change the active subspace.

Collaborative Tools. The second evaluation was concerned with the collaborative space and the attached tools. Users tested communication channels. Voice- and text-based exchanges were performed and were appreciated. For instance, the telepointer and its use to share documents was also welcomed. However, adults and young engineer reactions were different: for the first group, chat and live talk were more used before and during experiments. For young people exchanges were mainly concerned with simulations/real experiments results. Maybe cultural gap exists between the two categories and ones are more used with known tools (e.g., MSN or Skype) and our offer was not a part of their usual tools yet.

Realism and Interaction with Real Devices. This aspect was the most accepted. Indeed, users tried a lot to control remotely the real devices as well as the simulated ones. One could explain this by the playful nature of the interactions. Fortunately, we verified that after the discovery phase, users found the principle very comfortable: they can work at any time from anywhere. In addition, the proposed virtual environment was considered as enough realistic (mainly devices representation) to let users work as if they were near the device, and the video feedback increases this feeling.

5.2. Knowledge and Skills Acquisition. This part of the evaluation is the most difficult. Indeed, it was impossible for us to compare between the classical approach and the new one: many bias exist. For instance, we noticed that people spend more time when using SyTroN than when they are in a real classroom (2 times more). Also, exchanges between students are more frequent. In fact the problem is more general, and we are not equipped to tackle it. This issue may be addressed through cognitive and neurosciences approaches. We started to do it in a more global context, namely, human-machine interfaces objective evaluation with the hope to derive some generic tools allowing to evaluate learning processes.

\section{Conclusions}

We detailed the SyTroN concept, the approaches we developed to design its architecture, the components we built, its use as a functional platform, and some preliminary evaluations. First, we strongly concentrate our work on automatic control aspects (closing high-/low- speed loops over Internet) which are not described here. The second step was to predesign the virtual desk and its components. Voice, video and written messages channels were integrated within the system. A central system managing technical and administrative matters finishes our core developments. We have an operational system which can constitute a good basis for further improvements like adding an Intelligent Tutoring System (ITS) to assist automatically teachers. Indeed, teachers currently act in an old fashion by accompanying learners. An ITS can help to personalize more the relationship between each partner. The other contribution we made and which must be also improved in the future is the use of real devices to let learners confront to real problems.

Recently, we started experimenting the use of SyTroN in maintenance and dismantlement missions preparation in nuclear power plants [19]. The matter here is not to learn but to prepare stressing and dangerous missions. The key question for such systems remains the efficiency and the way to evaluate it. This is an open problem, and recent advances in neurosciences and cognitive sciences will help to find solutions and thus give objective tools in designing Elearning systems.

\section{Acknowledgments}

The authors would like to acknowledge to Amelie Imafouo. SyTroN can also be tested at http://www.emn .fr/x-auto/reposit.

\section{References}

[1] C. A. Lynch, "The transformation of scholarly communication and the role of the library in the age of networked information," The Serials Librarian, vol. 23, no. 3-4, pp. 5-20, 1993.

[2] F. Crison, A. Lécuyer, D. M. d'Huart, J.-M. Burkhardt, G. Michel, and J.-L. Dautin, "Virtual technical trainer: learning how to use milling machines with multi-sensory feedback in virtual reality," in Proceedings of IEEE Virtual Reality Conference (VR '05), pp. 139-145, Bonn, Germany, March 2005.

[3] S. Gerbaud, N. Mollet, F. Ganier, B. Arnaldi, and J. Tisseau, "GVT: a platform to create virtual environments for procedural training," in Proceedings of IEEE Virtual Reality Conference (VR '08), pp. 225-232, Reno, Neb, USA, March 2008.

[4] S. Gerbaud, N. Mollet, and B. Arnaldi, "Virtual environments for training: from individual learning to collaboration with humanoids," in Technologies for E-Learning and Digital Entertainment, vol. 4469 of Lecture Notes in Computer Science, pp. 116-127, Springer, Berlin, Germany, 2007.

[5] B. Lepori, L. Cantoni, and C. Succi, "The introduction of elearning in european universities: models and strategies," in Digitaler Campus, M. Kerres and B. Voss, Eds., pp. 74-83, 
Vom Medienprojekt zum nachhaltigen Medieneinsatz in der Hochschule, Berlin, Germany, 2003.

[6] V. Kukk, "Analysis of experience: fully web-based introductory course in electrical engineering," in Proceedings of the 1st International Workshop on E-Learning and Virtual and Remote Laboratories (VIRTUAL-LAB '04), pp. 111-118, Setúbal, Portugal, August 2004.

[7] S. Graf and B. List, "An evaluation of open source elearning platforms stressing adaptation issues," in Proceedings of the 5th IEEE International Conference on Advanced Learning Technologies (ICALT '05), pp. 163-165, Kaohsiung, Taiwan, July 2005.

[8] J. Trevelyan, "A framework for understanding engineering practice," in Proceedings of the ASEE Annual Conference and Exposition, pp. 1-20, Pittsburgh, Pa, USA, June 2008.

[9] A. Balestrino, A. Bicch, A. Caiti, et al., "From tele-laboratory to e-learning in automation curricula at the university of pisa," in Proceedings of the 16th IFAC World Congress (IFAC '05), Praga, Czech Republic, July 2005.

[10] A. Bicchi, A. Caiti, L. Pallottino, and G. Tonietti, "On-line robotic experiments for tele-education at the University of Pisa," International Journal of Robotic Systems, vol. 22, no. 4, pp. 217-230, 2004.

[11] D. Kim, K. Choi, S. Lee, C. Jeon, and J. Yoo, "Implementation of a web-based hybrid educational system for enhancing learning efficiency of engineering experiments," in Technologies for E-Learning and Digital Entertainment, vol. 4469 of Lecture Notes in Computer Science, pp. 357-368, Springer, Berlin, Germany, 2007.

[12] D. Gillet, F. Geoffroy, K. Zeramdini, A. V. Nguyen, Y. Rekik, and Y. Piguet, "The cockpit: an effective metaphor for web-based experimentation in engineering education," International Journal of Engineering Education, vol. 19, no. 3, pp. 389-397, 2003.

[13] R. B. Sepe Jr. and N. Short, "Web-based virtual engineering laboratory (VE-LAB) for collaborativeexperimentation on a hybrid electric vehicle starter/alternator," IEEE Transactions on Industry Applications, vol. 36, no. 4, pp. 1143-1150, 2000.

[14] G. Charpak, La Main à La Pâte. Les Sciences a L'Ecole Primaire, Flammarion, Paris, France, 1996.

[15] L. E. Carlson and J. F. Sullivan, "Hands-on engineering: learning by doing in the integrated teaching and learning program," International Journal of Engineering Education, vol. 15, no. 1, pp. 20-31, 1999.

[16] T. R. Harris, J. D. Bransford, and S. P. Brophy, "Roles for learning sciences and learning technologies in biomedical engineering education: a review of recent advances," Annual Review of Biomedical Engineering, vol. 4, pp. 29-48, 2002.

[17] W. Winn, "The impact of three-dimensional immersive virtual environments on modern pedagogy," Tech. Rep. HITL R-9715, Human Interface Technology Laboratory, Seattle, Wash, USA, 1997.

[18] E. Cuddihy and D. Walters, "Embodied interaction in social virtual environments," in Proceedings of the 3rd International Conference on Collaborative Virtual Environments (CVE '00), pp. 181-188, San Francisco, Calif, USA, September 2000.

[19] L. Brayda, L. Rossi, L. Taverna, and R. Chellali, "Collaborative virtual environments for sharing product lifecycle," in Proceedings of 2nd International Virtual Manufacturing Workshop (VIRMAN '08), Turin, Italy, October 2008. 

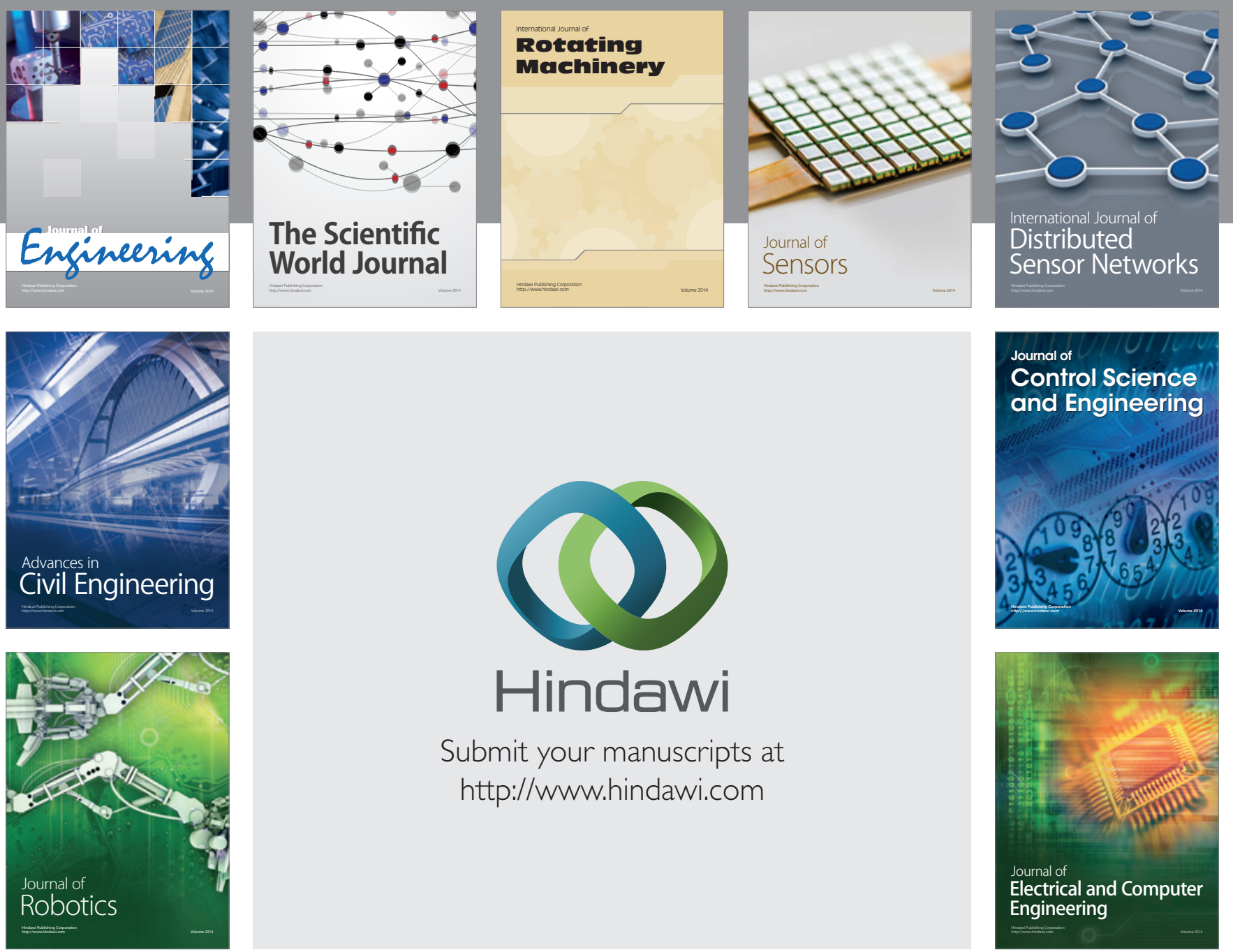

Submit your manuscripts at

http://www.hindawi.com
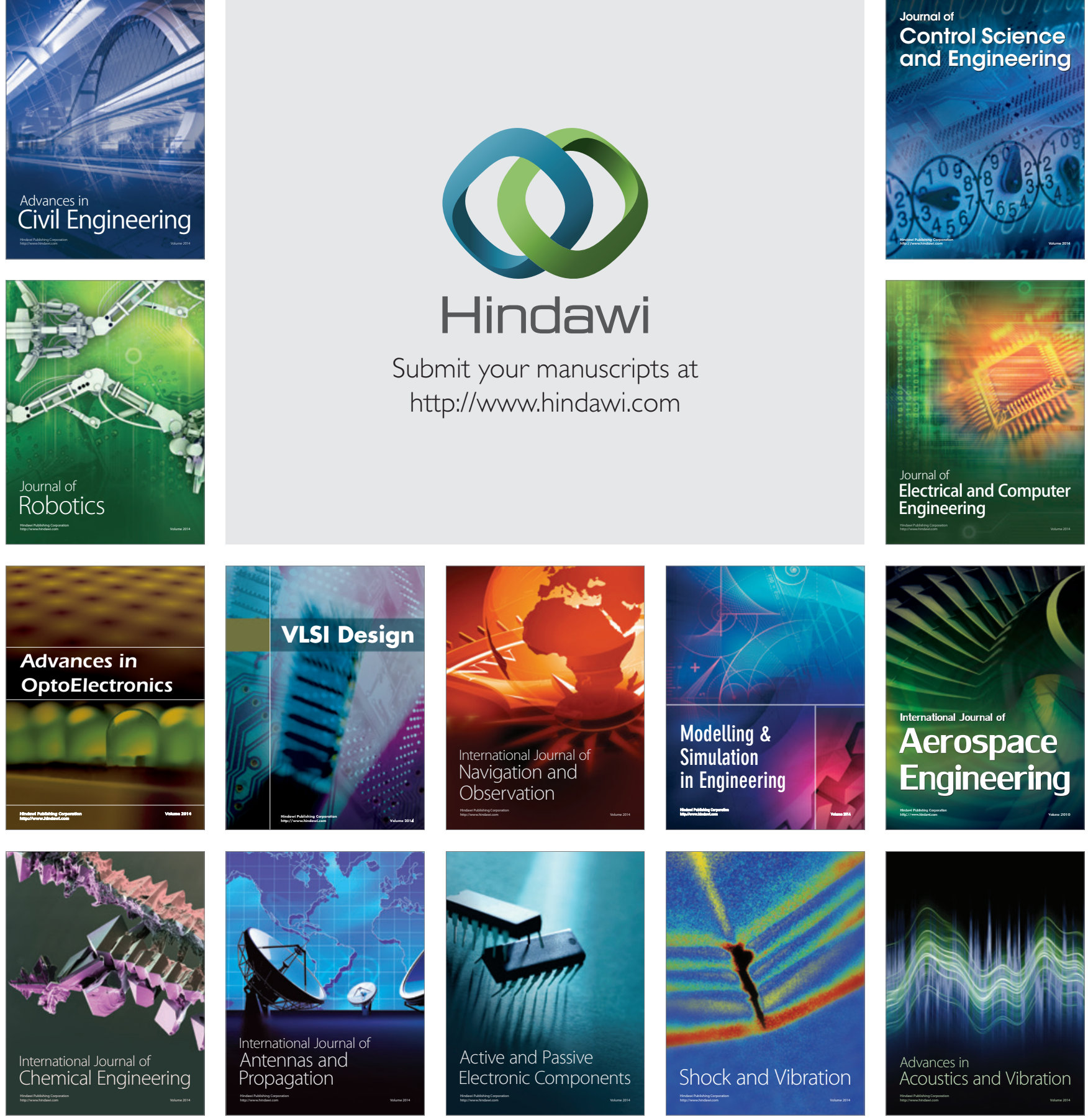